\title{
Soluble angiogenesis markers in gastric tumor patients
}

\author{
Piotr Mysliwiec ${ }^{1}$, Krystyna Pawlak², Roman Bandurski'1, Bogusław Kedra ${ }^{1}$ \\ $12^{\text {nd }}$ Department of General and Gastroenterological Surgery, ${ }^{2}$ Department of Nephrology \\ and Transplantology, Medical University of Białystok, Białystok, Poland
}

\begin{abstract}
Gastric cancer is the second commonest cause of cancer-associated death in the world. Its molecular markers can be useful not only for diagnostic, but also prognostic purposes. The aim of the study was to assess the usefulness of soluble angiogenesis markers such as endoglin and VEGFR2 in gastric cancer patients and to compare these results with those of VEGF levels. As a secondary objective, we compared the concentrations of all three soluble markers in plasma and serum. The study was performed on 26 patients with gastric cancer (17 intestinal-type and 9 diffuse-type), and additionally in 2 patients with B cell lymphoma and 2 with gastro-intestinal stromal tumor. In summary, we showed increases in circulating VEGF-A in patients with both types of gastric cancer. The levels of VEGFR2 did not change significantly in patients with gastric cancer as compared to healthy subjects. Interestingly, after the operation greater levels of VEGFR2 were observed in patients without metastases. Both VEGF and VEGFR2 circulating levels were greater in patients with lymphoma, when compared to both gastric cancer patients and the control group. However, because of small number of patients, this requires further studies. Presented data suggests that endoglin does not seem to be a valuable tool in the assessment of gastric cancer invasion and spread.
\end{abstract}

Key words: gastric cancer, angiogenesis, VEGF-A, VEGFR2, endoglin

\section{Introduction}

Gastric cancer is the second commonest cause of cancer-associated death in the world [1]. Although surgical treatment in early stages may offer cure of the disease, many patients present with micrometastases. In such situation, chemotherapy might be warranted [2]. Molecular markers of solid tumors, if accessible, can not only be useful in classifying the cancer, but they can also suggest dissemination of the disease [3].

Neo-angiogenesis is of crucial importance for gastric cancer growth and nutrition, with vascular endothelial growth factor (VEGF) being the most important growth factor involved in the process [4]. VEGF plays an important role in progression, invasion and spread of several gastrointestinal cancers, including gastric cancer. The tissue expression of VEGF can be associated with lymph node metastases and indicated poor prognosis [5]. Because of positive correlation between blood concentration of VEGF and the stage of gastric cancer [6], there have been trials to inhibit angiogenesis in gastric cancer [7].

Correspondence: P. Mysliwiec, Bacieczki 68A, 15-685 Białystok; tel.: (+48) 503085569, fax.: (+4885) 7468622, e-mail: mpiotr@umwb.edu.pl
VEGF-A, which is the most important form of VEGF, acts via its two receptors, VEGFR1 (or Flt-1) and VEGFR2 (or KDR) [8]. They are expressed almost exclusively in the endothelium of vessels lining or penetrating solid tumors [9]. VEGFR2 has critical functions in physiological and pathological angiogenesis [8]. The expression of VEGFR2 in intestinal-type gastric cancer was found to correlate with the vessel count and the stage of disease [10]. There is evidence that VEGFR2 plays a key role in regulation of proliferation of gastric cancer cells [11]. A soluble form of VEGFR2 was also described, with potential to inhibit angiogenesis [12]. So far no data are available on soluble VEGFR2 in gastric cancer.

One of the recently described substances important for angiogenesis is endoglin [5]. Endoglin, also known as CD105, is a trans-membrane co-receptor for TGF$\beta 1$ and TGF- $\beta 3$. It modulates the cell response to those mediators. Endoglin is induced by hypoxia. It is present mainly in new vessels. Therefore it is very useful for assessment of neo-angiogenesis of malignant neoplasms [13]. Endoglin assessed in gastric cancer tissues using immunohistochemistry was shown to correlate with newly formed blood vessels [14].

Endoglin is expressed not only on cell surface, since its soluble form can be detected also in blood [15]. Until 
now, there are no sufficient data on endoglin in blood of patients with gastric cancer

The aim of our study was to assess the usefulness of soluble angiogenesis markers such as endoglin and VEGFR2 in gastric cancer patients and to compare these results with those of VEGF levels. As a secondary objective, we compared the concentrations of all three soluble markers in plasma and serum. We also assessed the levels of all molecules in few patients with non-cancerous gastric tumors: lymphomas and gastro-intestinal stromal tumors.

\section{Materials and methods}

Patients. The studies were performed on plasma and serum samples from 30 patients with gastric tumors treated in the II Department of General and Gastroenterological Surgery, Medical University of Bialystok. This group comprised 26 patients with gastric cancer (17 intestinal-type - Lauren I and 9 diffuse-type - Lauren II), lymphoma (B cell lymphoma) in 2 patients and gastro-intestinal stromal tumor (GIST) in 2. The group of patients with gastric tumors was characterized in Table 1.

The control group consisted of 24 apparently healthy subjects. It comprised 13 men and 11 women, aged 32 to 78 (mean 64,9). These were apparently healthy subjects. Patients and volunteers with clinical or laboratory signs of infection were excluded from the study. The study was approved by the local ethical committee.

sPlasma and Serum samples. In all patients, samples were collected before surgery and at post-operative day 10. After overnight fasting, $4.5 \mathrm{ml}$ venous blood was poured into a tube. After clot formation, specimens were centrifuged at $1000 \mathrm{rpm}$ for 15 minutes in $4^{\circ} \mathrm{C}$. The supernatant - serum was transferred to Eppendorf's tube and frozen in $-70^{\circ} \mathrm{C}$ until determination. For plasma collection, tubes with $0.5 \mathrm{ml}$ EDTA were used. Immediately after sampling, specimens were centrifuged at $1000 \mathrm{rpm}$ for 15 minutes in $4^{\circ} \mathrm{C}$. The supernatant - platelet poor plasma was transferred to Eppendorf's tube and frozen at $-70^{\circ} \mathrm{C}$ until analysis.

Plasma and serum samples were used for ELISA determinations. VEGF-A, sVEGFR2 and endoglin were assessed using commercially available ELISA kits purchased from Research Diagnostics Inc., USA.

Statistical analysis. Statistical analysis. Shapiro-Wilk test was used for assessing normality. Kruskal-Wallis ANOVA, MannWhitney, Wilcoxon and Spearman's tests were used accordingly. Results are given as median; full range (minimum, maximum). A value of $p<0.05$ was regarded as significant. As there were only two patients with both lymphoma or GIST, no analysis was performed for those groups.

\section{Results}

In our study, we treated values of VEGF-A $<4 \mathrm{pg} / \mathrm{ml}$ as below detection range. This was the case in 9 out of 24 control subjects. We found a significant increase in VEGF-A concentration in plasma of the patients with both intestinal-type and diffuse-type gastric cancer (type I and II according to Lauren) ( $\mathrm{p}<0.05$ for both) (Fig. 1), as compared to the control group. The level of VEGF did not differ significantly between the two subgroups of gastric cancer patients. In the two
Table 1. Clinical and pathological characteristics of the gastric tumor patients

\begin{tabular}{|c|c|c|}
\hline & Patient no. & $(\%)$ \\
\hline Gender: & & \\
\hline Male & 20 & $(66.7)$ \\
\hline Female & 10 & (33.3) \\
\hline Tumor site: & & \\
\hline Stomach body & 14 & $(46.7)$ \\
\hline Antrum & 10 & (33.3) \\
\hline Subcardial region & 6 & $(20)$ \\
\hline Operation type: & & \\
\hline Total gastrectomy & 14 & $(46.7)$ \\
\hline Subtotal gastrectomy & 9 & $(30)$ \\
\hline Partial gastrectomy & 2 (GIST) & $(6.67)$ \\
\hline Laparotomy & 5 & $(16.7)$ \\
\hline Resection type: & & \\
\hline Curative & 11 & $(36.7)$ \\
\hline Palliative & 19 & $(63.3)$ \\
\hline $\begin{array}{l}\text { lumor infiltration (gastric cancer } \\
\text { patients only): }\end{array}$ & & \\
\hline PTl & 4 & (15.4) \\
\hline PT2 & 1 & $(3.85)$ \\
\hline PT3 & 6 & $(23.1)$ \\
\hline PT4 & 15 & $(57.7)$ \\
\hline $\begin{array}{l}\text { Iymph node status (gastric cancer } \\
\text { patients only): }\end{array}$ & & \\
\hline PNO & 7 & $(26.9)$ \\
\hline PN1 & 2 & $(7.69)$ \\
\hline PN2 & 3 & (11.5) \\
\hline PN3 & 14 & (53.8) \\
\hline $\begin{array}{l}\text { Metastases status (gastric cancer patients } \\
\text { only): }\end{array}$ & & \\
\hline PM0 & 13 & $(50)$ \\
\hline PMl & 13 & $(50)$ \\
\hline $\begin{array}{l}\text { Grade of differentiation (gastric cancer } \\
\text { patients only): }\end{array}$ & & \\
\hline $\mathrm{G} 2$ & 8 & $(30.8)$ \\
\hline G3 & 18 & $(69.2)$ \\
\hline
\end{tabular}

patients with gastric lymphoma VEGF level was over 6-fold greater than in other groups of patients.

As can be seen in Fig. 2, VEGFR2 showed a tendency towards similar changes as VEGF-A, however without significant differences. We found a positive correlation between preoperative VEGF-A and VEGFR2 values in gastric cancer patients (Spearman's $\mathrm{R}=0.42$, with $\mathrm{p}<0.05$ ). The highest levels (but not exceeding 2-fold increase) were observed in the two lymphoma patients. In the two patients with GIST the 


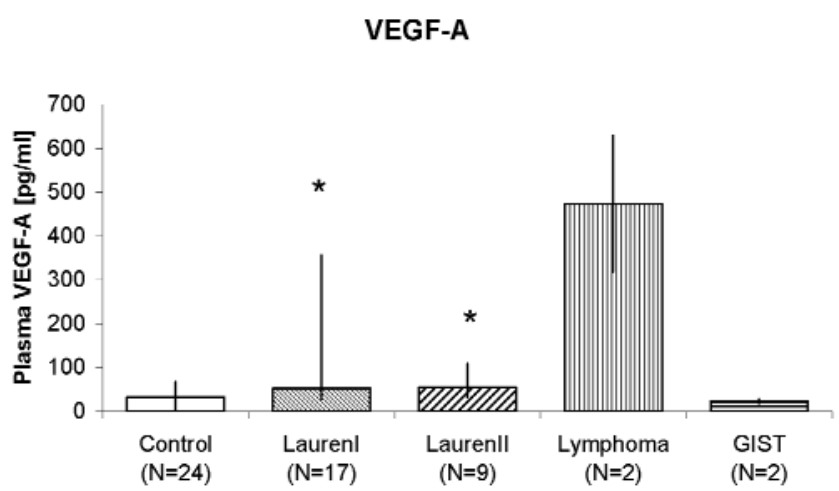

Fig. 1. Preoperative concentration of VEGF-A in plasma of normal controls (control), and patients with different gastric tumors: gastric cancer of type I according to Lauren (Lauren I), gastric cancer of type II according to Lauren (Lauren II), lymphoma and gastrointestinal stromal tumor (GIST). Results are given as median, full range. $*$ - significant difference from control group at $\mathrm{p}<0.05$. For Lymphoma and GIST groups no statistical analysis was performed ( $\mathrm{N}=2$ in both groups).

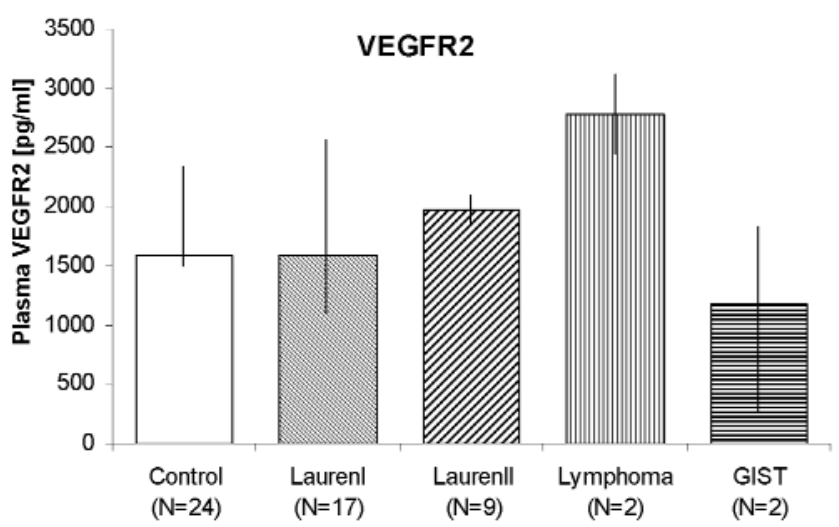

Fig. 2. Preoperative concentration of VEGF-R2 in plasma of normal controls (control), and patients with different gastric tumors: gastric cancer of type I according to Lauren (Lauren I), gastric cancer of type II according to Lauren (Lauren II), lymphoma and gastrointestinal stromal tumor (GIST). Results are given as median, full range. No significant differences were found. For Lymphoma and GIST groups no statistical calculation was performed $(\mathrm{N}=2$ in both groups).

plasma concentrations of VEGFR2 were lower than those of the control group or gastric cancer patients.

Endoglin concentrations in plasma of the patients with gastric tumors and those in the control group did not differ significantly (Fig. 3).

When comparing plasma and serum levels of the markers assessed in gastric cancer patients (Fig. 4), we found much greater values of VEGF in serum than in plasma, with a 3-fold difference for Lauren II type $(p<0.01)$ and 10 -fold for Lauren I type $(p<0.001)$. There was a high positive correlation between plasma and serum VEGF (Spearman's $\mathrm{R}=0.76$ with $\mathrm{p}<0.05$, data not shown). As far as VEGFR2 is concerned, the values did not change significantly whether assessed in plasma or in serum (Fig. 5). The median values of

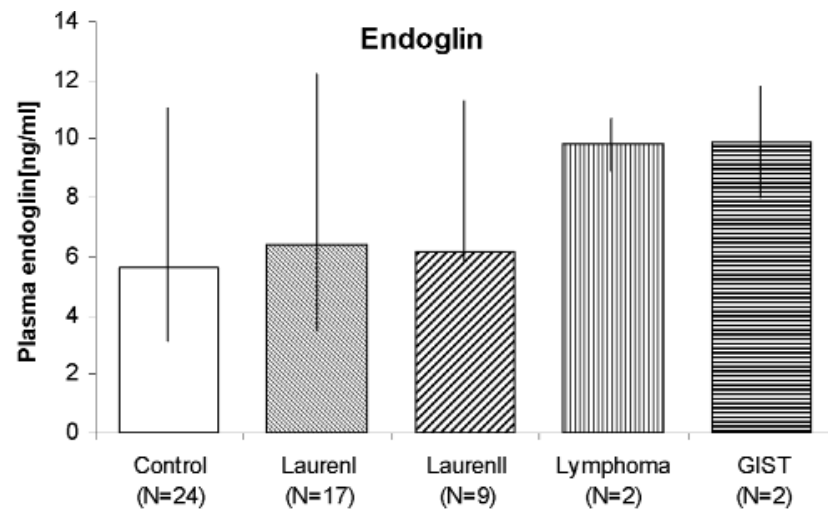

Fig. 3. Preoperative concentration of endoglin in plasma of normal controls (control), and patients with different gastric tumors: gastric cancer of type I according to Lauren (Lauren I), gastric cancer of type II according to Lauren (Lauren II), lymphoma and gastrointestinal stromal tumor (GIST). Results are given as median, full range. No significant differences were found. For Lymphoma and GIST groups no statistical calculation was performed.

endoglin obtained in plasma samples tended to be about $20 \%$ lower than those found in serum, but the difference was not significant (Fig. 6). There was a high positive correlation between the values obtained for plasma and serum samples (Spearman's $\mathrm{R}=0.82$ with $\mathrm{p}<0.05$ - data not shown).

Operation resulted in a marked increase in VEGF in the plasma of both gastric cancer groups as compared to the preoperative values $(\mathrm{p}<0,05$ for Lauren I and Lauren II.) (Fig. 7). A similar tendency was observed for patients with Lymphoma and GIST.

We did not observe any significant changes in VEGFR2 concentrations before and 10 days after surgical treatment (Fig. 8). However, when comparing postoperative VEGFR2 in metastasis-positive and metastasis-negative patients, we found markedly greater values in the latter subgroup $(p<0.001-$ data not shown).

In contrast to VEGF, postoperative endoglin concentrations in serum of gastric cancer patients dropped significantly $(p<0,05)$ for Lauren I type (Fig. 9). No other differences were observed.

We did not find any significant correlations between VEGF, VEGFR2 or endoglin in plasma or serum and any clinical or pathological parameters of gastric cancer stage in our patients.

\section{Discussion}

Angiogenesis is a key process leading to gastric cancer growth, invasion and dissemination. Among numerous substances involved in new vessel formation, VEGF is one of the best characterized [16]. VEGF expression enhancement has been previously demonstrated in gastric cancer patients when compared to normal controls. An association of VEGF serum concentrations 


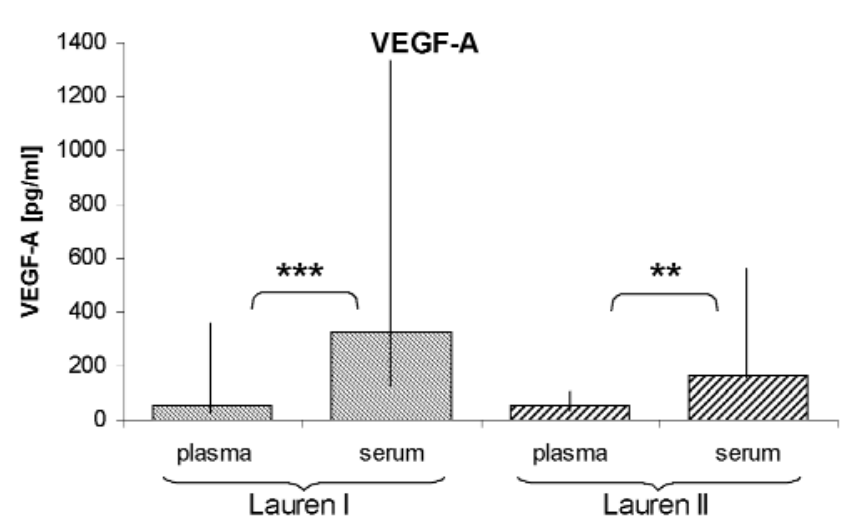

Fig. 4. Preoperative concentration of VEGF-A in plasma and in serum of the patients with gastric cancer of type I according to Lauren (Lauren I), and of type II according to Lauren (Lauren II). Results are given as median, full range. Significant differences are marked with $* *_{-} \mathrm{p}<0.01, * * *_{\text {-p }}<0.001$.

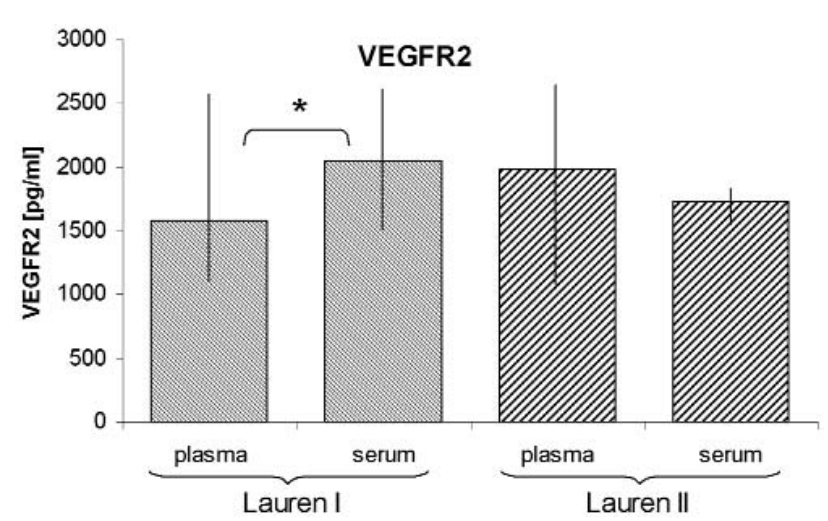

Fig. 5. Preoperative concentration of sVEGF-R2 in plasma and in serum of the patients with gastric cancer of type I according to Lauren (Lauren I), and of type II according to Lauren (Lauren II). Results are given as median, full range. Significant differences are marked with *- $<<0.05$.

with tumor grade and size were also reported [17]. It was also shown that VEGF may play a role in peritoneal recurrence of gastric cancer [18].

Our results show an increase in plasma VEGF-A in both intestinal-type and diffuse-type gastric cancer patients when compared with healthy subjects (Fig. 1). This is in accordance with previous publications reporting that VEGF-A levels in both plasma and serum were greater in gastric cancer patients than in the control group [19].

After the operation, at postoperative day 10, we observed a significant increase in VEGF levels in both groups of patients with gastric cancer (Fig. 4). Ding et al. [19] assessed VEGF-A levels 3 weeks after the operation and found a decrease in VEGF-A concentrations. Different times of blood sampling may at least explain partly the difference with our study. There is evidence that in oxidative stress results in substantial increases in VEGF-A production [20]. This might

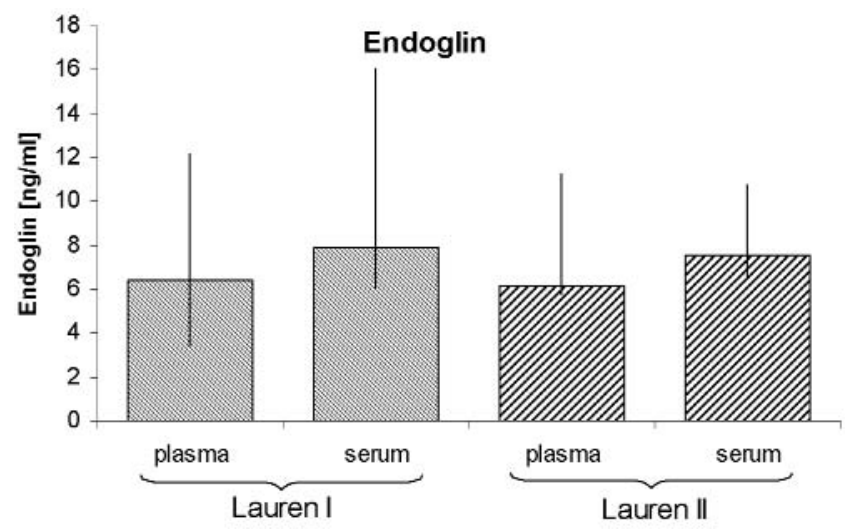

Fig. 6. Preoperative concentration of endoglin in plasma and in serum of the patients with gastric cancer of type I according to Lauren (Lauren I), and of type II according to Lauren (Lauren II). Results are given as median, full range. No significant differences were found.

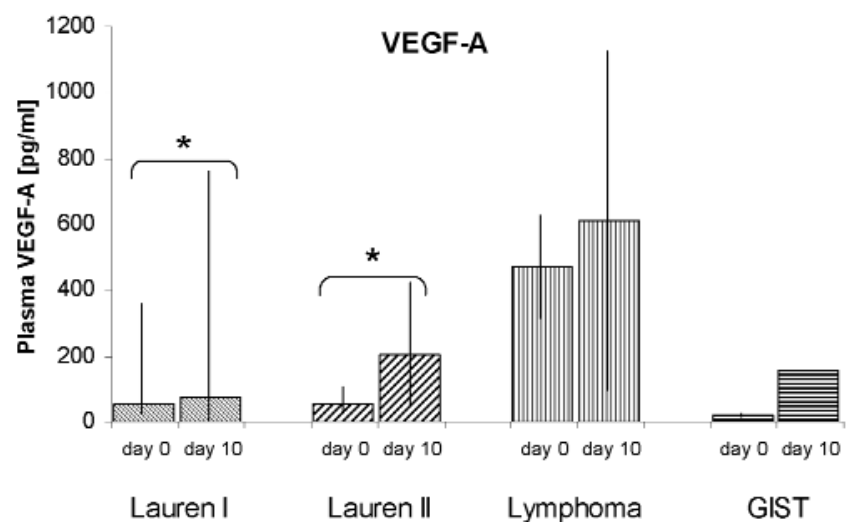

Fig. 7. Influence of operation on the concentration of VEGF-A in plasma of patients with different gastric tumors: gastric cancer of type I according to Lauren (Lauren I), gastric cancer of type II according to Lauren (Lauren II), lymphoma and gastrointestinal stromal tumor (GIST). Results are given as median, full range. Significant differences are marked with $*_{-} p<0.05$. For Lymphoma and GIST groups no statistical calculation was performed $(\mathrm{N}=2$ in both groups).

explain the rise in VEGF-A on postoperative day 10. It has also been demonstrated that level of serum VEGF after the operation depends on radicality of resection [17]. It falls in patients after radical gastrectomy and rises after palliative operation. Unfortunately in our study, most patients were operated at an advanced stage of the disease and the majority of operations were palliative.

When comparing the values of soluble angiogenesis markers studied in plasma and serum of patients with gastric cancer, we found significantly greater values of VEGF-A in serum than in plasma (Fig. 4). Our results confirm findings by Ding et al. [19]. We observed a high positive correlation between plasma and serum values when paired results were compared. We suggest that especially VEGF-A in gastric cancer patients should be assessed in serum rather than in plasma. 


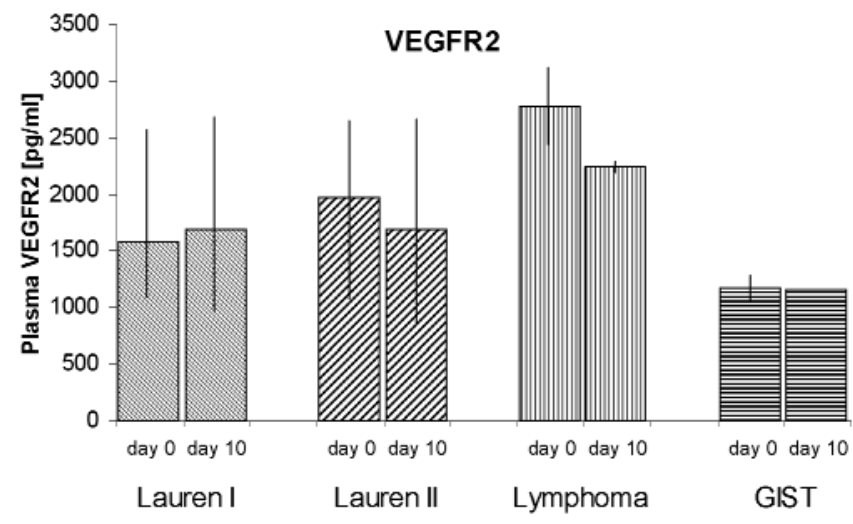

Fig. 8. Influence of operation on the concentrations of sVEGF-R2 in serum of patients with different gastric tumors: gastric cancer of type I according to Lauren (Lauren I), gastric cancer of type II according to Lauren (Lauren II), lymphoma and gastrointestinal stromal tumor (GIST). Results are given as median, full range. No significant differences were found. For Lymphoma and GIST groups no statistical analysis was performed ( $\mathrm{N}=2$ in both groups)

We also observed, which has not yet been demonstrated before, an increase in VEGF-A in the patients with other stomach tumors: GIST and lymphoma (Fig. 1). However, as there were only two patients with either type of non-cancerous gastric neoplasms included in our study, these findings need confirmation. It has been reported that VEGF is strongly expressed in diffuse large B cell nodal lymphomas [21]. The level of circulating VEGF in blood of patients with leukaemia or non-Hodgkin's lymphoma was described as a predictor of poor prognosis [22]. VEGF has even been suggested as a potential target for antiangiogenetic treatment of aggressive lymphomas [21].

VEGFR2 is one of two types of tissue receptors for VEGF-A [8]. A soluble form of VEGFR2, which circulates in blood, can bind to VEGF-A and so act as an inhibitor of angiogenesis [12,23]. Several studies have confirmed that inhibition of VEGFR2 leads to growth reduction of human neoplasms [24,25]. Until now, no data on soluble VEGFR2 in gastric cancer have been available. We did not demonstrate any significant change in circulating VEGFR2 in gastric cancer patients, when compared to healthy subjects (Fig. 2). However, we found a marked difference between VEGFR2 in plasma of metastases-negative and -positive gastric cancer, as assessed postoperatively $(\mathrm{p}<0.001)$. Patients with distant metastases had lower levels of VEGFR2 as assessed on day 10 postoperatively. We speculate that after tumor resection, the only focus of cancer left corresponds to metastases. As we show in the present study, VEGF-A level rised after the operation. As VEGF has been reported to play an important role in aggressive malignancies, its increase might be greater in patients with distant metastases. Also in our patients, postoperative VEGF-A level in
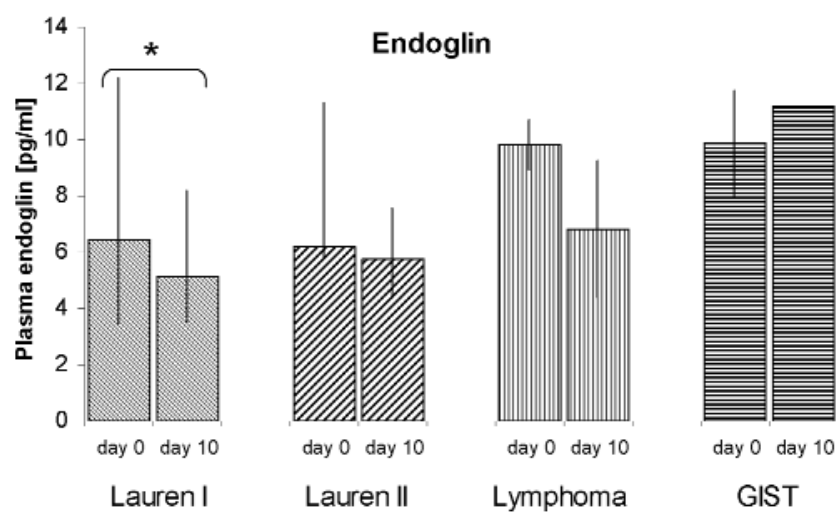

GIST

Fig. 9. Influence of operation on the concentration of endoglin in serum of patients with different gastric tumors: gastric cancer of type I according to Lauren (Lauren I), gastric cancer of type II according to Lauren (Lauren II), lymphoma and gastrointestinal stromal tumor (GIST). Results are given as median, full range. Significant difference is marked with $*_{-} \mathrm{p}<0.05$. For Lymphoma and GIST groups no statistical analysis was performed $(\mathrm{N}=2$ in both groups).

plasma tended to be greater in metastasis-positive than in metastasis-negative patients (median 130 versus $100 \mathrm{ng} / \mathrm{ml}$ ), though the difference was not significant. VEGFR2 might be bound to greater amounts of circulating VEGF-A and so - decrease in the circulation. The importance of our finding for gastric cancer patients remains to be established.

Comparative analysis of VEGFR2 concentrations in plasma and serum showed greater values in serum in patients with intestinal-type gastric cancer (Lauren I) (Fig. 5). This was not demonstrated for diffuse-type cancer group.

Endoglin, a receptor for transforming growth factor $\beta 1$ and $\beta 3$ is expressed predominantly on newly formed microvessels. It is crucial for angiogenesis by modulating endothelial-mesenchymal signalling and thus enhancing proliferation [26]. Endoglin is also claimed to be involved in such phenomena as cell adhesion and migration [27]. Soluble endoglin has been reported to rise in breast cancer patients who developed metastases, as compared to both normal controls and cancer patients without distant spread [28]. Elevated serum endoglin was associated with distant metastases not only in breast but also colorectal and other solid tumors [15]. Until now there were no reports on endoglin levels in serum in patients with gastric cancer.

Our results did not demonstrate any significant differences in endoglin concentrations in plasma of patients with gastric cancer (Fig. 3). We observed however greater values of endoglin in the patients with rare gastric tumors: lymphoma and GIST. As mentioned above, this finding needs further confirmation in larger groups of patients. Interestingly, endoglin concentrations dropped postoperatively in plasma of 
patients with intestinal type (Lauren I) gastric cancer and had a similar tendency in diffuse-type cancer (Fig. 9). The possible explanation might be the removal of the tumor.

The assessment of endoglin in plasma and serum showed a tendency for greater serum levels (Fig. 6), though the difference was not significant.

We failed to demonstrate a correlation between endoglin and the stage of gastric cancer. This might be attributed to the difference between tissue expression of this angiogenic compound and the level of its circulating form.

In summary, we showed increases in circulating VEGF-A in patients with both types of gastric cancer (Lauren I and II). The levels of VEGFR2 did not change significantly in patients with gastric cancer as compared to healthy subjects. Interestingly, after the operation greater levels of VEGFR2 were observed in patients without metastases. Both VEGF and VEGFR2 circulating levels were greater in patients with lymphoma, when compared to both gastric cancer patients and the control group. However, because of small number of patients, this requires further studies. Presented data suggests that endoglin does not seem to be a valuable tool in the assessment of gastric cancer invasion and spread.

\section{References}

[ 1] Leung WK, Sung JJ. Chemoprevention of gastric cancer. Eur J Gastroenterol Hepatol. 2006;18;867-871.

[2] Hohenberger P, Gretschel S. Gastric cancer. Lancet 2003; 362;305-315.

[3] Lin W, Kao HW, Robinson D et al. Tyrosine kinases and gastric cancer. Oncogene. 2000;19;5680-5689.

[4] Liu DH, Zhang XY, Fan DM et al. Expression of vascular endothelial growth factor and its role in oncogenesis of human gastric carcinoma. World J Gastroenterol. 2001;7;500505.

[5] Nikiteas NI, Tzanakis N, Theodoropoulos G et al. Vascular endothelial growth factor and endoglin (CD-105) in gastric cancer. Gastric Cancer. 2007;10;12-17.

[ 6] Lissoni P, Malugani F, Bonfanti A et al. Abnormally enhanced blood concentrations of vascular endothelial growth factor (VEGF) in metastatic cancer patients and their relation to circulating dendritic cells, IL-12 and endothelin-1. J Biol Regul Homeost Agents. 2001;15;140-144.

[ 7] Lazar D, Raica M, Sporea I et al. Tumor angiogenesis in gastric cancer. Rom J Morphol Embryol. 2006;47;5-13.

[ 8] Shibuya M, Claesson-Welsh L. Signal transduction by VEGF receptors in regulation of angiogenesis and lymphangiogenesis. Exp Cell Res. 2006;312;549-560.

[ 9] Barleon B, Siemeister G, Martiny-Baron G et al. Vascular endothelial growth factor up-regulates its receptor fms-like tyrosine kinase 1 (FLT-1) and a soluble variant of FLT-1 in human vascular endothelial cells. Cancer Research. 1997;57; 5421-5425.

[10] Takahashi Y, Cleary KR, Mai M et al. Significance of vessel count and vascular endothelial growth factor and its receptor (KDR) in intestinal-type gastric cancer. Clin Cancer Res. $1996 ; 2 ; 1679-1684$.
[11] Ren J, Dong L, Xu CB et al. The role of KDR in the interactions between human gastric carcinoma cell and vascular endothelial cell. World J Gastroenterol. 2002;8;596-601.

[12] Kou B, Li Y, Zhang L et al. In vivo inhibition of tumor angiogenesis by a soluble VEGFR-2 fragment. Exp Mol Pathol. 2004;76;129-137.

[13] Duff SE, Li C, Garland JM et al. CD105 is important for angiogenesis: evidence and potential applications. FASEB J. 2003; 17;984-992.

[14] Yu JX, Zhang XT, Liao YQ et al. Relationship between expression of CD105 and growth factors in malignant tumors of gastrointestinal tract and its significance. World $J$ Gastroenterol. 2003;9;2866-2869.

[15] Takahashi N, Kawanishi-Tabata R, Haba A et al. Association of serum endoglin with metastasis in patients with colorectal, breast, and other solid tumors, and suppressive effect of chemotherapy on the serum endoglin. Clin Cancer Res. 2001; 7;524-532.

[16] Yonemura Y, Endo Y, Tabata K et al. Role of VEGF-C and VEGF-D in lymphangiogenesis in gastric cancer. Int $J$ Clin Oncol. 2005;10;318-327.

[17] Karayiannakis AJ, Syrigos KN, Polychronidis A et al. Circulating VEGF levels in the serum of gastric cancer patients: correlation with pathological variables, patient survival, and tumor surgery. Ann Surg. 2002;236;37-42.

[18] Aoyagi K, Kouhuji K, Yano S et al. VEGF significance in peritoneal recurrence from gastric cancer. Gastric Cancer. 2005;8;155-163.

[19] Ding S, Lin S, Dong X et al. Potential prognostic value of circulating levels of vascular endothelial growth factor-A in patients with gastric cancer. In Vivo. 2005;19;793-795.

[20] Schafer G, Cramer T, Suske G et al. Oxidative stress regulates vascular endothelial growth factor-A gene transcription through Sp1- and Sp3-dependent activation of two proximal GC-rich promoter elements. J Biol Chem. 2003;278;81908198.

[21] Tzankov A, Heiss S, Ebner S et al. Angiogenesis in nodal B cell lymphomas: a high throughput study. J Clin Pathol. 2007;60;476-482.

[22] Moehler TM, Ho AD, Goldschmidt $\mathrm{H}$ et al. Angiogenesis in hematologic malignancies. Crit Rev Oncol Hematol. 2003;45; 227-244.

[23] Hasan J, Jayson GC. VEGF antagonists. Expert Opin Biol Ther. 2001;1;703-718.

[24] Lang SA, Brecht I, Moser C et al. Dual inhibition of Raf and VEGFR2 reduces growth and vascularization of hepatocellular carcinoma in an experimental model. Langenbecks Arch Surg. 2008;393;333-341.

[25] Lu N, Gao Y, Ling Y et al. Wogonin suppresses tumor growth in vivo and VEGF-induced angiogenesis through inhibiting tyrosine phosphorylation of VEGFR2. Life Sci. 2008;82;956963.

[26] Fonsatti E, Maio M. Highlights on endoglin (CD105): from basic findings towards clinical applications in human cancer. J Transl Med. 2004;2;18.

[27] Guo B, Rooney P, Slevin M et al. Overexpression of CD105 in rat myoblasts: Role of CD105 in cell attachment, spreading and survival [In Process Citation]. Int J Oncol. 2004;25;285291.

[28] Li C, Guo B, Wilson P et al. Plasma levels of soluble CD105 correlate with metastasis in patients with breast cancer. Int $J$ Cancer. 2000;89;122-126.

Submitted: 18 June, 2008 Accepted after reviews: 26 October, 2008 